\title{
Polyakov loop, Hadron Resonance Gas Model and Thermodynamics of QCD ${ }^{1}$
}

\author{
E. Megías*, E. Ruiz Arriola ${ }^{\dagger}$ and L.L. Salcedo ${ }^{\dagger}$ \\ ${ }^{*}$ Grup de Física Teòrica and IFAE, Departament de Física, Universitat Autònoma de Barcelona, Bellaterra \\ E-08193 Barcelona, Spain \\ ${ }^{\dagger}$ Departamento de Física Atómica, Molecular y Nuclear and Instituto Carlos I de Física Teórica y \\ Computacional, Universidad de Granada, E-18071 Granada, Spain
}

\begin{abstract}
We summarize recent results on the hadron resonance gas description of QCD. In particular, we apply this approach to describe the equation of state and the vacuum expectation value of the Polyakov loop in several representations. Ambiguities related to exactly which states should be included are discussed.
\end{abstract}

Keywords: finite temperature, QCD thermodynamics, heavy quarks, chiral quark models, Polyakov loop

PACS: 11.10.Wx, 11.15.-q, 11.10.Jj, 12.38.Lg, 11.30, 12.38.-t

\section{INTRODUCTION}

The thermodynamics of Quantum Chromodynamics (QCD) has received much attention due to the possible existence of new phases of nuclear matter at sufficiently high temperatures and densities, such as the quark-gluon plasma [1]. The phase transition from a confined to a deconfined phase is characterized by a change of order parameters connected with different symmetries of the system. The relevant degrees of freedom in the deconfined phase are quarks and gluons, while in the confined phase these constituents form color singlet bound states and mostly resonances, i.e. hadrons and possibly glueballs. When physical quark masses are considered, recent lattice simulations indicate that this transition is actually a hadron-quark-gluon crossover [2].

The Hadron Resonance Gas (HRG) model is a useful approach to describe the thermodynamics of QCD in the confined phase. It is based on the assumption that physical observables in this phase admit a representation in terms of hadronic states which are treated as non-interacting and point-like particles [3]. These states are usually taken as the conventional hadrons listed in the review by the Particle Data Group (PDG) [4], so the completeness of the PDG states is assumed. A commonly used order parameter for the hadron-quark-gluon crossover is the Polyakov loop in the fundamental representation, as it is related to the free energy of a heavy quark " $h$ " placed in a thermal medium $[5,6,7]$,

$$
L_{\mathbf{3}}=\left\langle\operatorname{trP} e^{i g \int_{0}^{1 / T} A_{0} d x_{0}}\right\rangle \simeq e^{-F_{h} / T},
$$

where $\mathrm{P}$ indicates path ordering and $A_{0}$ is the Euclidean time component of the gluon field. While the HRG model has been traditionally applied to study the equation of state of QCD, a similar hadronic representation for the Polyakov loop in the fundamental representation was recently formulated in [8]. This approach has been confronted with recent lattice data and confirmed the accuracy of the model for temperatures in the range $150 \mathrm{MeV}<T<190 \mathrm{MeV}[8,7]$. The Polyakov loop in any other representation can be computed in lattice QCD, and the existence of Casimir scaling relations has been guessed from lowest order perturbation theory $[9,10]$. The generalization of the hadronic representation to other representations besides the fundamental one is a natural step forward which could be used to test the existence of exotic states in the QCD spectrum. In this communication we briefly overview the HRG model, and study its realization within a particular quark model. We advance some results for the Polyakov loop in the representations 8, 10, 15 and 27.

\footnotetext{
${ }^{1}$ Invited plenary talk given by E. Megías at the XXXVI Brazilian Workshop on Nuclear Physics, 1-5 September 2013, Maresias, São Sebastião, São Paulo, Brazil.
} 


\section{QCD AT FINITE TEMPERATURE AND SYMMETRIES}

QCD is accepted nowadays as the fundamental theory of strong interactions. The QCD Lagrangian

$$
\mathscr{L}_{\mathrm{QCD}}=\frac{1}{2} \operatorname{tr}\left(G_{\mu v}^{2}\right)+\sum_{f} \bar{q}_{f}\left(i \gamma_{\mu} D_{\mu}-m_{f}\right) q_{f}, \quad G_{\mu v}=\partial_{\mu} A_{v}-\partial_{v} A_{\mu}-i g\left[A_{\mu}, A_{v}\right],
$$

describes the dynamics of quarks $q$ and gluons $A_{\mu}$, which are the fundamental fields in the theory. $\mathscr{L}_{\mathrm{QCD}}$ is constructed to be invariant under color gauge transformations. The QCD equation of state can be derived from the partition function

$$
Z_{\mathrm{QCD}}=\operatorname{Tr} e^{-H / T}=\int \mathscr{D} A_{\mu, a} \exp \left[-\frac{1}{4} \int d^{4} x\left(G_{\mu \nu}^{a}\right)^{2}\right] \operatorname{Det}\left(i \gamma_{\mu} D_{\mu}-m_{f}\right) .
$$

Within the imaginary time formalism of finite temperature field theory, in the path integral of Eq. (3) the bosonic and fermionic fields obey periodic and anti-periodic boundary conditions in the Euclidean time, respectively, with periodicity $\beta=1 / T$. These boundary conditions are preserved under periodic gauge transformations.

In the massless quarks limit $m_{f}=0$, the theory is invariant at the classical level under scale transformations. This symmetry is broken by quantum corrections through the inevitable regularization, leading to a non-zero value for the interaction measure or trace anomaly $\varepsilon-3 P$. In this limit, $\mathscr{L}_{\mathrm{QCD}}$ is also invariant under chiral transformations, which are spontaneously broken by the chiral condensate in the vacuum $\langle\bar{q} q\rangle \neq 0$. At high enough temperature, i.e. for $T>T_{\chi}$, the chiral symmetry is restored, and this is signaled by a vanishing value of $\langle\bar{q} q\rangle$.

In the opposite limit of infinitely heavy quarks $m_{f} \rightarrow \infty$, there is a larger symmetry related to the center $\mathbb{Z}\left(N_{c}\right)$ of the gauge group, as gauge transformations which are periodic in Euclidean time modulo an element of the center are allowed, i.e. $\Lambda\left(\vec{x}, x_{0}+\beta\right)=z \Lambda\left(\vec{x}, x_{0}\right), z^{N_{c}}=1$. In the Polyakov gauge $\partial_{0} A_{0}=0$, an example of such a transformation is $\Lambda\left(x_{0}\right)=e^{i 2 \pi x_{0} \lambda /\left(N_{c} \beta\right)}$, where $\lambda=\operatorname{diag}\left(1,1, \ldots, 1-N_{c}\right)$, under which the Polyakov loop transforms as

$$
L_{\mathbf{3}}=\left\langle\operatorname{tr}_{c} e^{i g A_{0} / T}\right\rangle \longrightarrow e^{i 2 \pi / N_{c}} L_{\mathbf{3}} .
$$

From Eq. (4) it follows that $L_{3}$ vanishes in the $\mathbb{Z}\left(N_{c}\right)$ symmetric phase. This means that the heavy quark free energy diverges, corresponding then to a confined phase. Likewise a non $\mathbb{Z}\left(N_{c}\right)$ symmetric phase is characterized by a nonvanishing value of the Polyakov loop, leading to finite free energy which corresponds to a deconfined phase. So the Polyakov loop can be used as an order parameter for the confinement-deconfinement phase transition in QCD in the limit $m_{f} \rightarrow \infty$. The picture that emerges is that in QCD there are two phase transitions: one related to chiral symmetry, and another one related to confinement (or center symmetry), with transition temperatures $T_{\chi}$ and $T_{D}$ respectively, which are quark-mass dependent. In the real world, because of finite quark masses, the chiral condensate and the Polyakov loop are approximate order parameters only, and lattice simulations predict $T_{\chi} \approx T_{D} \approx 200 \mathrm{MeV}[5,11]$. The physical mechanism behind the close agreement between both transitions remains unclear, a situation that worsens for finite quark chemical potential, where lattice simulation are on more shaky grounds.

\section{HADRON RESONANCE GAS MODEL AND EQUATION OF STATE OF QCD}

While in the deconfined phase of QCD the quarks and gluons are liberated to form a plasma, in the confined/chiral symmetry broken phase the relevant degrees of freedom are hadronic states. The idea of the HRG model is to describe the equation of state of QCD in terms of a free gas of hadrons $[3,12,13,14,15,16,17,18,19,20]$,

$$
\frac{1}{V} \log Z=-\int \frac{d^{3} p}{(2 \pi)^{3}} \sum_{\alpha} \zeta_{\alpha} g_{\alpha} \log \left(1-\zeta_{\alpha} e^{-\sqrt{p^{2}+M_{\alpha}^{2}} / T}\right)
$$

with $g_{\alpha}$ the degeneracy factor, $\zeta_{\alpha}= \pm 1$ for bosons and fermions respectively, and $M_{\alpha}$ the hadron mass. The Hagedorn formula for the trace anomaly follows from Eq. (5) and the standard thermodynamic relations. It writes

$$
\frac{\varepsilon-3 P}{T^{4}}=\sum_{k=1}^{\infty} \int d M\left(\frac{\partial n_{m}(M)}{\partial M}+(-1)^{k+1} \frac{\partial n_{b}(M)}{\partial M}\right) \frac{1}{2 k \pi^{2}}\left(\frac{M}{T}\right)^{3} K_{1}\left(k \frac{M}{T}\right),
$$

where $K_{1}(z)$ refers to the modified Bessel function. $n_{m}$ and $n_{b}$ are the cumulative numbers of mesons and baryons (including antibaryons), defined as $n(M)=\sum_{\alpha} g_{\alpha} \Theta\left(M-M_{\alpha}\right)$, where $\Theta$ is the step function. $n(M)$ represents the 

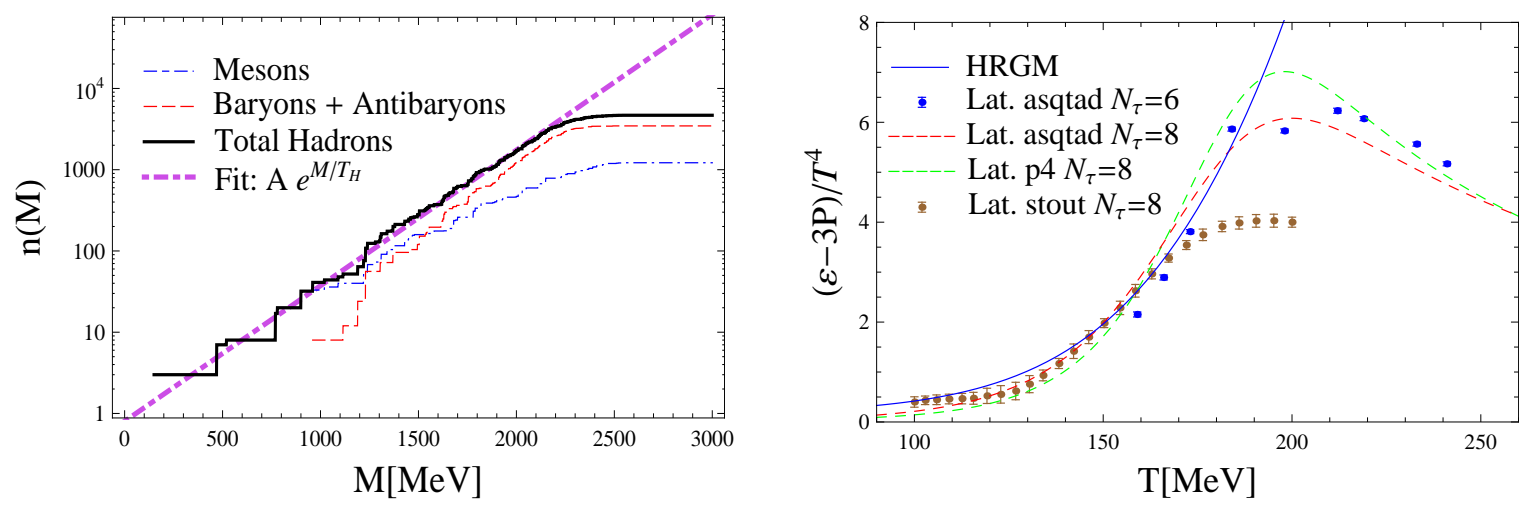

FIGURE 1. Left: Cumulative number $n$ as a function of the hadron mass $M$ (in MeV), for hadrons with $u, d$ and $s$ quarks, computed in the RQM $[21,22]$. Right: Trace anomaly $(\varepsilon-3 P) / T^{4}$ as a function of temperature (in MeV). We compare lattice data for asqtad and p4 actions [11] (after temperature downshift of $T_{0}=15 \mathrm{MeV}$ ) and stout action [5], with the HRG model computed with the RQM spectrum shown in the left figure.

number of hadrons with mass less than $M$. Hagedorn proposed that the cumulative number of hadrons in QCD is approximately given by $n(M) \simeq A e^{M / T_{H}}$, where $T_{H}$ is the so called Hagedorn temperature. We show in Fig. 1 (left) the cumulative number of hadrons with $u, d$ and $s$ quarks computed in the Relativized Quark Model (RQM) [21, 22] up to a cutoff $M \approx 2300 \mathrm{MeV}$. The total cumulative number can be approximated to the exponential form with $A=0.80$, $T_{H}=260 \mathrm{MeV}$ and $\chi^{2} / \mathrm{dof}=0.031$, in the regime $500 \mathrm{MeV}<M<2300 \mathrm{MeV}$. The spectrum obtained can be used to compute the trace anomaly within the HRG approach given by Eq. (6). The result and its comparison with lattice data shows that the HRG model gives a good description of the trace anomaly for $T<180 \mathrm{MeV}$, see Fig. 1 (right).

\section{HADRON RESONANCE GAS MODEL FOR THE POLYAKOV LOOP}

The expectation value of the Polyakov loop in the irrepresentation $\mu$ of the color gauge group $\mathrm{SU}\left(N_{c}\right)$ is given by

$$
L_{\mathrm{QCD}, \mu}(T):=\frac{Z_{\mathrm{QCD}, \mu}}{Z_{\mathrm{QCD}}}
$$

where $Z_{\mathrm{QCD}}$ is the physical partition function obtained by projecting onto states which are singlet at every point, and $Z_{\mathrm{QCD}, \mu}$ is the partition function with a static color charge in the irrep $\mu$. In the confined phase the static source (heavy quark or gluon) is screened by dynamical quarks and gluons from the medium to form a heavy hadron (or glueball). Within the present approach, we retain the confining forces that give rise to the hadron, but neglect the interaction of this hadron to other dynamical hadrons present in the resonance gas, i.e. non-confining forces. This assumption is parallel to that of the HRG model for the partition function.

\section{Results in the fundamental representation}

Based on these considerations, we have shown in [8] that a hadronic representation of the Polyakov loop in the fundamental representation $(\mu=\mathbf{3})$ is given by

$$
L_{\mathbf{3}}(T) \approx \sum_{\alpha} g_{h \alpha} e^{-\Delta_{h \alpha} / T}, \quad \Delta_{h \alpha}=M_{h \alpha}-m_{h},
$$

where $g_{h \alpha}$ are the degeneracies and $\Delta_{h \alpha}$ are the masses of hadrons with exactly one heavy quark (the mass of the heavy quark itself $m_{h}$ being subtracted). A natural step is to check to what extent this hadronic sum rule is fulfilled by experimental states compiled in the PDG [4]. Single charmed hadrons are preferable, as there are more available data than single bottomed hadrons. Specifically, when considering the lowest-lying single-charmed mesons and baryons with $u, d$, and $s$ as the dynamical flavors, i.e. a total of 12 meson states and 42 baryon states, we find that the result 

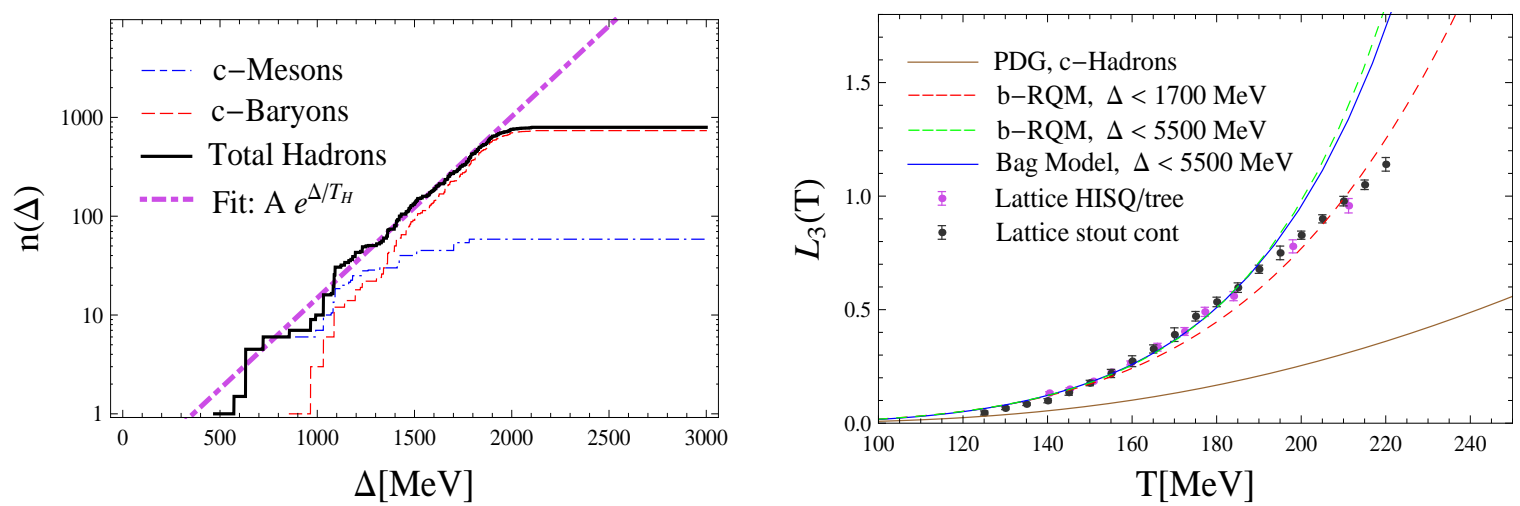

FIGURE 2. Left: Cumulative number $n$ as a function of the heavy quark subtracted hadron mass $\Delta=M-m_{h}$ (in $\mathrm{MeV}$ ), for the hadron spectrum with a $c$ quark and light dynamical quarks $u, d$ and $s$, computed in the RQM [21, 22]. Right: Polyakov loop in the fundamental representation as a function of $T$ (in MeV). Lattice data from the HISQ/tree action [19] and stout action [5] are displayed. We compare the HRG model result when including in Eq. (8) the lowest-lying charmed hadrons from PDG [4], the RQM spectrum with one $b$ quark and cut-off $\Delta<1700 \mathrm{MeV}, \Delta<5500 \mathrm{MeV}$, and the MIT bag model with cut-off $\Delta<5500 \mathrm{MeV}$ [8].

falls short to saturate the sum rule, cf. Fig. 2 (right). The conclusion is that one needs many more states than for the trace anomaly $[8,23,7]$. This suggests using quark model spectra for $[h \bar{q}]$ and $[h q q]$ color singlet states with one heavy $h=c, b$ quark and the remaining light quarks $q=u, d, s$. This study was performed in [8,24]. The hadron spectrum obtained with the RQM is displayed in Fig. 2 (left), while we show in Fig. 2 (right) the Polyakov loop computed from Eq. (8). The result using the MIT bag model [25] is also displayed. Now the sum rule is almost saturated.

It remains the important question of completeness of hadronic states, and the possible existence of exotic states as well as their contribution on the HRG model side of the sum rule. We will give some insights in the next sections.

\section{Ambiguities in the hadron resonance gas approach}

Within the present approximation, there is some ambiguity as to exactly which states should be included in the sum rule Eq. (8). The problem is as follows, let $V$ be the spatial neighborhood of the static color source with the dynamical constituents (quarks, antiquarks, gluons). The procedure of just adding constituents in $V$, to form color singlets with the source, and computing the resulting spectrum, will certainly produce states which are spurious. Namely, states composed of a genuine heavy hadron plus one or more ordinary dynamical hadrons. One can consider two possible prescriptions to remove the spurious states (see Ref. [26] for details):

- Include in the sum rule just configurations of constituents which are color irreducible, i.e., without subclusters of constituents forming a color singlet by themselves,

$$
L_{\mu}(T):=\sum_{i, \text { irred }} g_{i} e^{-\beta \Delta_{i}}
$$

- Cancel the reducible configurations by considering the quotient

$$
\tilde{L}_{\mu}(T):=\frac{Z_{\mu}(T)}{Z_{1}(T)}, \quad \text { where } \quad Z_{\mu}(T):=\sum_{i, \text { all }} g_{i} e^{-\beta \Delta_{i}} .
$$

$Z_{\mu}(T)$ contains all (reducible and irreducible) configurations of type $\mu$, and $Z_{1}(T)$ is the physical partition function. $Z_{\mu}(T)$ factorizes in two contributions: the partition function of the neighborhood $V$ of the static source times a hadron gas with a hole in $V$.

Both prescriptions will be studied in the next section within a particular quark model. As we will see, the cancellation of reducible configurations with the denominator in Eq. (10) only happens for non exotic states. 


\section{CONSTITUENT QUARK MODELS AND THE HADRON RESONANCE GAS}

An effective approach to the physics of QCD at finite temperature is provided by chiral quark models coupled to gluon fields in the form of a Polyakov loop [27, 28, 29, 30, 31, 32, 33, 34, 35, 36]. While most of these works remain within a mean field approximation, we have shown in [29] that such approximation erases information such as the Polyakov loop expectation values in higher representations. We show in this section that the sum rule for the fundamental Polyakov loop Eq. (8) is fulfilled in these models only when one advocates the local and quantum nature of the Polyakov loop. The need of these corrections was stressed in [29, 30, 32, 33, 37]. Other irreps will be explored as well.

\section{The Polyakov-Constituent Quark Model}

We consider a model that describes QCD using free constituent dynamical quarks and gluons with a Polyakov variable $\Omega(\mathbf{x})$ at each point of space. The partition function of the model is given by [29, 33, 38, 39]

$$
Z_{\mathrm{PCM}}=\int \prod_{\mathbf{x}} d \Omega(\mathbf{x}) e^{-S_{\mathrm{PCM}}(\Omega, T)},
$$

where the matrix $\Omega(\mathbf{x})$ is kept as a quantum and local degree of freedom. $d \Omega(\mathbf{x})$ is the invariant $\mathrm{SU}\left(N_{c}\right)$ group integration measure $\left(N_{c}=3\right)$ at the point $\mathbf{x}$. The action splits as a sum of contributions from each kind of constituent, and it reads

$$
S_{\mathrm{PCM}}(\Omega, T)=\sum_{c=q, \bar{q}, g} S_{c}(\Omega, T), \quad \text { where } \quad S_{c}(\Omega, T)=g_{c} \zeta_{c} \int \frac{d^{3} x d^{3} p}{(2 \pi)^{3}} \operatorname{tr} \log \left(1-\lambda \zeta_{c} \Omega_{c}(\mathbf{x}) e^{-E_{c} / T}\right) .
$$

The action depending on the quarks $S_{q}+S_{\bar{q}}$ is obtained from the corresponding fermion determinant, and $S_{g}$ mimics gluodynamics [40]. $E_{c}=\sqrt{\mathbf{p}^{2}+M_{c}^{2}}$ is the energy of the constituent $c$, and $M_{c}$ is the corresponding constituent mass. The degeneracy factors are $g_{q, \bar{q}}=2 N_{f}$ and $g_{g}=2$. In the notation of Eq. (12), $\Omega_{c}$ is the Polyakov loop in the fundamental $(c=q)$, antifundamental $(c=\bar{q})$ or adjoint $(c=g)$ representation, so we can equally use the standard notation $\Omega_{\mathbf{3}}, \Omega_{\overline{3}}$ and $\Omega_{\mathbf{8}}$ respectively. $\lambda$ is a parameter that counts the number of constituents. One can always replace $\lambda \rightarrow 1$. After a series expansion in Eq. (12), the Lagrangian density for constituent $c$ reads

$$
\mathscr{L}_{c}(\mathbf{x})=-T g_{c} \zeta_{c} \sum_{n=1}^{\infty} \frac{\left(\lambda \zeta_{c}\right)^{n}}{n} J_{n}\left(M_{c}, T\right) \operatorname{tr}\left(\Omega_{c}^{n}(\mathbf{x})\right), \quad c=q, \bar{q}, g
$$

(see $[39,41,26]$ for an alternative expansion) where we have defined $J_{n}\left(M_{c}, T\right):=\int \frac{d^{3} p}{(2 \pi)^{3}} e^{-n E_{c} / T} \sim e^{-n M_{c} / T}$ for $T \ll M_{c}$, displaying the statistical Boltzmann factor characteristic of multi-quark or multi-gluon states [29]. For instance, meson-like contributions induce corrections of the form $\sim e^{-2 M_{q} / T}$, and baryon-like contributions behave as $\sim e^{-N_{c} M_{q} / T}$. To take into account quantum corrections in the Polyakov loop, an integration in the color group must be performed [29]. In addition, there are local effects associated to the correlation of Polyakov loops. We assume that the space is decomposed into domains of size $V_{\sigma}$, such that two Polyakov loops are fully correlated if they lie within the same domain and are fully uncorrelated otherwise. The contribution to the partition function of any such domain is $\int d \Omega e^{-\frac{V_{\sigma}}{T} \sum_{c} \mathscr{L}_{c}}$. The volume rule $V_{\sigma}=8 \pi T^{3} / \sigma^{3}$ has been motivated in $[29,33]$ to describe the crossover, although its performance at low temperatures remains to be analyzed.

\section{Expansion in the number of constituents}

We can perform an expansion of observables in the number of constituents. When using the estimate given by Eqs. (9) for the Polyakov loop in the fundamental representation, one finds within the confined domain approximation

$$
\begin{aligned}
L_{\mathrm{PMC}, 3}= & \lambda \bar{Q}_{1}+\lambda^{2} \frac{1}{2}\left(Q_{1}^{2}+Q_{2}+2 G_{1} \bar{Q}_{1}\right)+\lambda^{3}\left(Q_{1}^{2}+\bar{Q}_{1} G_{1}\right) G_{1} \\
& +\lambda^{4}\left[\frac{1}{4}\left(5 Q_{1}^{2} G_{1}^{2}-Q_{1}^{2} G_{2}-Q_{2} G_{1}^{2}+Q_{2} G_{2}\right)+\frac{1}{6}\left(5 G_{1}^{3}-3 G_{1} G_{2}-2 G_{3}\right) \bar{Q}_{1}\right]+O\left(\lambda^{5}\right), \\
\simeq & g_{q}[h \bar{q}]+\frac{g_{q}}{2}\left(g_{q}+1\right)\left[h q^{2}\right]+2 g_{q}[h \bar{q} g]+2 g_{q}^{2}\left[h q^{2} g\right]+4 g_{q}\left[h \bar{q} g^{2}\right]+\frac{g_{q}}{2}\left(9 g_{q}-1\right)\left[h q^{2} g^{2}\right]+4 g_{q}\left[h \bar{q} g^{3}\right]+\mathscr{O}\left(\lambda^{5}\right),(14)
\end{aligned}
$$



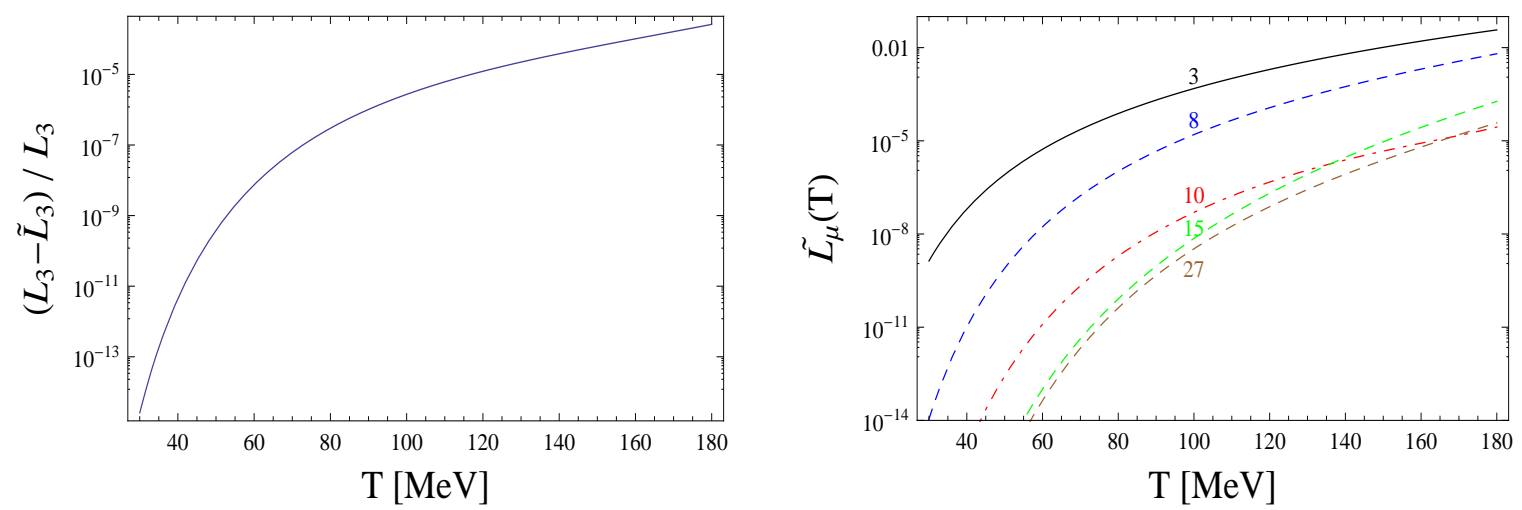

FIGURE 3. Left: Difference between $L_{\mathrm{PCM}, 3}$ and $\tilde{L}_{\mathrm{PCM}, 3}$ (normalized to $L_{\mathrm{PCM}, 3}$ ) as a function of $T$ (in $\mathrm{MeV}$ ), cf. Eqs. (14)-(15). Right: $\tilde{L}_{\mathrm{PCM}, \mu}$ as a function of $T$, for several irreps. From top to bottom $\mu=\mathbf{3 , 8}, \mathbf{1 0}, \mathbf{1 5}, \mathbf{2 7}$. In these plots we have included up to four constituents, used $N_{f}=2$, constituent quark and gluon masses $M_{q}=300 \mathrm{MeV}, M_{g}=664 \mathrm{MeV}$, and $\sigma=(425 \mathrm{MeV})^{2}$.

where in the second equality we have written the result in a schematic way. We have defined $Q_{n}(T)=g_{q} V_{\sigma} J_{n}\left(M_{q}, T\right)$ and $G_{n}(T)=g_{g} V_{\sigma} J_{n}\left(M_{g}, T\right)$, for quarks and gluons respectively. $\bar{Q}_{n}$ is numerically identical to $Q_{n}$ but it accounts for $n$ antiquarks. Each factor $Q_{n}, \bar{Q}_{n}$ or $G_{n}$ counts as $n$ quarks, antiquarks or gluons, respectively, corresponding to dynamical constituents from the medium which screen the Polyakov loop itself, the latter being identified with a heavy quark source " $h$ ". The factor in front of each term corresponds to the degeneracy. The first two terms $[h \bar{q}]$ and $\left[h q^{2}\right]$ are mesons and baryons, respectively, with a heavy quark and one or several light (anti)quarks. Quantization of the model would produce the energy levels $\Delta_{h \alpha}$ to be used in Eq. (8), as explained in [38]. This completes the connection with the HRG model for the Polyakov loop [8]. If the color cluster decomposition were exact, higher order terms could contain configurations that can be identified with hybrids, pentaquarks $\mathscr{O}\left(\lambda^{5}\right)$, etc. ${ }^{2}$ However, the situation is not so clear as we will see next. When using the second approach, Eq. (10), the result one gets is

$\tilde{L}_{\mathrm{PMC}, 3} \simeq \cdots-\frac{g_{q}^{2}}{6}\left(g_{q}+1\right)\left(g_{q}+2\right)\left[h q^{3} \bar{q}\right]-\frac{g_{q}}{24}\left(g_{q}+1\right)\left(g_{q}+2\right)\left(g_{q}+3\right)\left[h \bar{q}^{4}\right]+\frac{g_{q}}{2}\left(9 g_{q}-1\right)\left[h q^{2} g^{2}\right]+4 g_{q}\left[h \bar{q} g^{3}\right]+\mathscr{O}\left(\lambda^{5}\right)$,

where the terms up to $\mathscr{O}\left(\lambda^{3}\right)$ are identical to those in Eq. (14). The two approaches differ by terms of $\mathscr{O}\left(\lambda^{4}\right)$, and some of the configurations in $\tilde{L}_{\mathrm{PCM}, 3}$ at this order appear with negative weights, so the picture is certainly cleaner if just the irreducible configurations are retained. It follows that the ambiguity regarding color singlet clustering affects non-conventional hadrons only. The result for the Polyakov loop in the adjoint representation reads

$$
\begin{aligned}
\tilde{L}_{\mathrm{PCM}, \mathbf{8}} \simeq & 2\left[\Omega_{\mathbf{8}} g\right]+g_{q}^{2}[q \bar{q}]+4\left[\Omega_{\mathbf{8}} g^{2}\right]+\frac{g_{q}}{3}\left(g_{q}^{2}-1\right)\left(\left[\Omega_{\mathbf{8}} q^{3}\right]+\left[\Omega_{\mathbf{8}} \bar{q}^{3}\right]\right)+4 g_{q}^{2}\left[\Omega_{\mathbf{8}} q \bar{q} g\right]+4\left[\Omega_{\mathbf{8}} g^{3}\right]+4\left[\Omega_{\mathbf{8}} g^{4}\right] \\
& +\frac{g_{q}}{3}\left(g_{q}-1\right)\left(5 g_{q}+2\right)\left(\left[\Omega_{\mathbf{8}} q^{3} g\right]+\left[\Omega_{\mathbf{8}} \bar{q}^{3} g\right]\right)+9 g_{q}^{2}\left[\Omega_{\mathbf{8}} q \bar{q} g^{2}\right]+\mathscr{O}\left(\lambda^{5}\right)
\end{aligned}
$$

where $\Omega_{\mathbf{8}}$ is an adjoint source at rest, for instance two heavy quarks coupled adjointwise. In general, up to three constituents the two estimates coincide,

$$
L_{\mathrm{PCM}, \mu}=\tilde{L}_{\mathrm{PCM}, \mu}+O\left(\lambda^{4}\right), \quad \mu \leq \mathbf{2 7},
$$

and they differ by terms of $O\left(\lambda^{5}\right)$ for irreps beyond 27 [26]. We plot in Fig. 3 the Polyakov loop in several representations, computed with the PCM within the two different estimates of Eqs. (9) and (10). We display in the right figure only those irreps which lead to positive results in the regime depicted. Negative values are obtained for the representations $\mathbf{6}, \mathbf{1 5}^{\prime}$ and $\mathbf{2 4}$ at low temperatures, presumably due to an unrealistic behavior of $V_{\sigma}$ in that regime. A detailed study of these and other issues will be performed in a forthcoming paper [26].

\footnotetext{
${ }^{2}$ It is noteworthy that tetraquark states $\left[h \bar{q}^{2} q\right]$ are always reducible. When no confining interactions are switched off they split into two mesons $[h \bar{q}][\bar{q} q][26]$.
} 


\section{CONCLUSIONS}

The thermodynamics of the confined phase of QCD can be described in terms of a free gas of hadronic states. A similar approach is possible for the Polyakov loop in terms of color singlet states with a static source coupled to dynamical quarks and gluons. We have studied a particular realization of the HRG description by using a chiral quark model with Polyakov loop, in which the local and quantum nature of the Polyakov loop is taken into account. The HRG approach is however affected by ambiguities when a high enough number of dynamical constituents are coupled to the static source. A resolution of this puzzle will shed some light on the existence of exotic states in the QCD spectrum.

\section{ACKNOWLEDGMENTS}

Supported by Plan Nacional de Altas Energías (FPA2011-25948), DGI (FIS2011-24149), Junta de Andalucía grant FQM-225, Spanish Consolider-Ingenio 2010 Programme CPAN (CSD2007-00042), Spanish MINECO's Centro de Excelencia Severo Ochoa Program grant SEV-2012-0234, and the Juan de la Cierva Program.

\section{REFERENCES}

1. H. Meyer-Ortmanns, Rev.Mod.Phys. 68, 473-598 (1996), hep-lat/9608098.

2. Y. Aoki, G. Endrodi, Z. Fodor, S. Katz, and K. Szabo, Nature 443, 675-678 (2006), hep-lat / 0611014.

3. R. Hagedorn, Lect.Notes Phys. 221, 53-76 (1985).

4. J. Beringer, et al., Phys.Rev. D86, 010001 (2012).

5. S. Borsanyi, et al., JHEP 1009, 073 (2010), 1005.3508.

6. B. Lucini, and M. Panero, Phys.Rept. 526, 93-163 (2013), 1210.4997.

7. A. Bazavov, and P. Petreczky, Phys.Rev. D87, 094505 (2013), 1301.3943.

8. E. Megias, E. Ruiz Arriola, and L.L. Salcedo, Phys.Rev.Lett. 109, 151601 (2012), 1204.2424.

9. A. Dumitru, Y. Hatta, J. Lenaghan, K. Orginos, and R. D. Pisarski, Phys.Rev. D70, 034511 (2004), hep-th/0311223.

10. S. Gupta, K. Huebner, and O. Kaczmarek, Phys.Rev. D77, 034503 (2008), 0711.2251.

11. A. Bazavov, T. Bhattacharya, M. Cheng, N. Christ, C. DeTar, et al., Phys.Rev. D80, 014504 (2009).

12. V. Yukalov, and E. Yukalova, Phys.Part.Nucl. 28, 37-65 (1997).

13. J. Cleymans, and K. Redlich, Phys.Rev. C60, 054908 (1999), nucl-th/9903063.

14. N. O. Agasian, Phys. Lett. B519, 71-77 (2001).

15. A. Tawfik, Phys.Rev. D71, 054502 (2005).

16. E. Megias, E. Ruiz Arriola, and L.L. Salcedo, Phys.Rev. D80, 056005 (2009), 0903.1060.

17. P. Huovinen, and P. Petreczky, Nucl.Phys. A837, 26-53 (2010), 0912 . 2541.

18. S. Borsanyi, G. Endrodi, Z. Fodor, A. Jakovac, S. D. Katz, et al., JHEP 1011, 077 (2010), 1007.2580.

19. A. Bazavov, et al., Phys. Rev. D85, 054503 (2012), 1111.1710.

20. J. Noronha-Hostler, J. Noronha, and C. Greiner, Phys.Rev. C86, 024913 (2012), 1206.5138.

21. S. Godfrey, and N. Isgur, Phys. Rev. D32, 189-231 (1985).

22. S. Capstick, and N. Isgur, Phys.Rev. D34, 2809 (1986).

23. E. Ruiz Arriola, L.L. Salcedo, and E. Megias (2013), 1304.2245.

24. E. Megias, E. Ruiz Arriola, and L.L. Salcedo, Nucl.Phys.Proc.Suppl. 234, 313-316 (2013), 1207 . 7287.

25. A. Chodos, R. Jaffe, K. Johnson, C. B. Thorn, and V. Weisskopf, Phys.Rev. D9, 3471-3495 (1974).

26. E. Megias, E. Ruiz Arriola, and L.L. Salcedo, (in progress) (2013).

27. P. N. Meisinger, and M. C. Ogilvie, Phys.Lett. B379, 163-168 (1996), hep-lat/9512011.

28. K. Fukushima, Phys.Lett. B591, 277-284 (2004), hep-ph/ 0310121.

29. E. Megias, E. Ruiz Arriola, and L.L. Salcedo, Phys.Rev. D74, 065005 (2006), hep-ph/ 0412308.

30. E. Megias, E. Ruiz Arriola, and L.L. Salcedo, PoS JHW2005, 025 (2006), hep-ph 0511353.

31. C. Ratti, M. A. Thaler, and W. Weise, Phys.Rev. D73, 014019 (2006), hep-ph/ 0506234.

32. E. Megias, E. Ruiz Arriola, and L.L. Salcedo, AIP Conf.Proc. 892, 444-447 (2007), hep-ph/ 0610095.

33. E. Megias, E. Ruiz Arriola, and L.L. Salcedo, Phys.Rev. D74, 114014 (2006), hep-ph / 0607338.

34. B.-J. Schaefer, J. M. Pawlowski, and J. Wambach, Phys. Rev. D76, 074023 (2007), 0704 . 3234.

35. G. A. Contrera, D. Gomez Dumm, and N. N. Scoccola, Phys. Lett. B661, 113-117 (2008), 0711.0139.

36. S. K. Ghosh, T. K. Mukherjee, M. G. Mustafa, and R. Ray, Phys.Rev. D77, 094024 (2008), 0710.2790.

37. E. Megias, E. Ruiz Arriola, and L.L. Salcedo, Eur.Phys.J. A31, 553-556 (2007), hep-ph/ 0610163.

38. E. Ruiz Arriola, E. Megias, and L.L. Salcedo, AIP Conf.Proc. 1520, 185-190 (2013), 1207.4875.

39. E. Megias, E. R. Arriola, and L.L. Salcedo (2013), 1307 . 7523.

40. P. N. Meisinger, M. C. Ogilvie, and T. R. Miller, Phys.Lett. B585, 149-154 (2004), hep-ph/ 0312272.

41. C. Sasaki, and K. Redlich, Phys.Rev. D86, 014007 (2012), 1204 . 4330. 